\title{
NEW VARIABLE JET MODELS FOR HH 34
}

\author{
A. C. RaGa ${ }^{1}$, A. Rodríguez-González ${ }^{1}$, A. Noriega-Crespo ${ }^{2}$, And A. Esquivel ${ }^{1}$ \\ ${ }^{1}$ Instituto de Ciencias Nucleares, Universidad Nacional Autónoma de México, Ap. 70-543, 04510 México, D.F., Mexico \\ ${ }^{2}$ Spitzer Science Center, California Institute of Technology, CA 91125, USA \\ Received 2011 September 8; accepted 2011 November 25; published 2011 December 12
}

\begin{abstract}
We consider newly derived proper motions of the HH 34 jet to reconstruct the evolution of this outflow. We first extrapolate ballistic trajectories for the knots (starting from their present-day positions and velocities) and find that at $\sim 1000 \mathrm{yr}$ in the future most of them will merge to form a larger-mass structure. This mass structure will be formed close to the present-day position of the HH 34S bow shock. We then carry out a fit to the ejection velocity versus time reconstructed from the observed proper motions (assuming that the past motion of the knots was ballistic) and use this fit to compute axisymmetric jet simulations. We find that the intensity maps predicted from these simulations do indeed match reasonably well the [S II] structure of HH 34 observed in Hubble Space Telescope images.
\end{abstract}

Key words: circumstellar matter - Herbig-Haro objects - ISM: individual objects (HH34) - ISM: jets and outflows - stars: formation

Online-only material: color figures

\section{INTRODUCTION}

HH 34 (a Herbig-Haro object included in the catalog of Herbig 1974) stepped into the limelight with a paper by Reipurth et al. (1986). In that work, the authors showed that HH 34 has a remarkable, jet-like chain of aligned knots (extending $\sim 20^{\prime \prime}$ from a faint, young star) pointing approximately southward toward a remarkable, bow shock structure (HH 34S, at $\sim 100^{\prime \prime}$ from the outflow source). A northern bow shock (HH $34 \mathrm{~N}$, also at $\sim 100^{\prime \prime}$ from the source) was later discovered by Bührke et al. (1988). A "counterjet" knot chain (extending $\sim 20$ " to the $\mathrm{N}$ of the outflow source) was only observed much later (in the IR long-slit spectra of García López et al. 2008, and in the Spitzer images of Raga et al. 2011b). A "giant outflow" consisting of condensations extending out to $\sim 13^{\prime}$ from the outflow source along the two lobes was discovered by Bally \& Devine (1994).

Observations of the HH 34 outflow include images (e.g., Reipurth \& Heathcote 1992; Ray et al. 1996) and long-slit spectra (e.g., Heathcote \& Reipurth 1992; Podio et al. 2006). Proper motions of this outflow have been measured by Heathcote \& Reipurth (1992), Eislöffel \& Mundt (1992), Devine et al. (1997), and Reipurth et al. (2002). Models of the HH 34 outflow include "3D/2D bow shocks" (e.g., Raga 1986; Morse et al. 1992), axisymmetric variable jet simulations (e.g., Raga \& NoriegaCrespo 1998; Raga et al. 2002) and 3D variable+precessing jet simulations (Masciadri et al. 2002).

Relatively recently, a new set of Hubble Space Telescope (HST) images of a number of Herbig-Haro (HH) jets has been obtained (Hartigan et al. 2011). These images include an [S II] 6716+30 and an $\mathrm{H} \alpha$ image of $\mathrm{HH} 34$. Raga et al. (2011c) have used the new [S II] image (obtained in 2007.83) together with a previous image (1998.71; see Reipurth et al. 2002) to measure the proper motions of the knots along the HH 34 jet.

The resulting proper motion velocities can be used to reconstruct the time-dependent ejection velocity history of the outflow. Interestingly, this is the first time that proper motion velocities can be used to this effect because the larger errors in previously measured proper motions resulted in incoherent reconstructed ejection histories.

For example, Raga et al. (2002, see their Figure 1) took the proper motions of Reipurth et al. (2002) and could not reconstruct a systematic ejection velocity history. They therefore used the spatial velocities as a function of position derived by Raga \& Noriega-Crespo (1998) from radial velocities read on the printed [S II] position-velocity diagram of HH 34 of Heathcote \& Reipurth (1992). These radial velocities do lead to a coherent reconstructed ejection velocity time variability, but are highly uncertain in the region within $\sim 10^{\prime \prime}$ from the outflow source (corresponding to dynamical timescales of $\sim 100 \mathrm{yr}$ ), in which the HH 34 jet has very broad emission line profiles.

In the present Letter, we take the ejection velocity history reconstructed by Raga et al. (2011c) from their proper motion measurements and use it to compute the dynamics of HH 34. This ejection velocity history has the peculiarity of having an ejection velocity that grows over the past $\sim 400 \mathrm{yr}$ (resulting from increasing proper motion velocities for the knots closer to the outflow source), a property that was absent in the ejection velocities deduced (from radial velocities) by Raga \& NoriegaCrespo (1998).

We first compute a simple model, in which we consider equal mass, ballistic knots with the present positions, and velocities deduced by Raga et al. (2011c) for the HH 34 jet knots. Considering also a mass and momentum "knot merging law," we follow the time evolution of the ensemble of knots. This exercise is described in Section 2.

We then take the ejection velocity history deduced (assuming ballistic motions) from the proper motions of Raga et al. (2011c), and add the ejection velocities deduced by Raga \& NoriegaCrespo (1998) from the radial velocities directly upstream and downstream of the HH $34 \mathrm{~S}$ bow shock. To this ejection velocity history we fit a double-sinusoidal function. This fit is then used to compute axisymmetric numerical simulations of the flow, as described in Section 3.

Finally, the results are summarized in Section 4.

\section{BALLISTIC, KNOT-MERGING MODEL}

We first consider the knot positions and proper motion velocities of the chain of aligned knots along the HH 34 jet derived by Raga et al. (2011c). The positions $x$ (from the outflow source) on the plane of the sky and the spatial velocities $v_{j}=v_{x} / \cos \phi$ of the knots are given in Table 1 (where $v_{x}$ is the 


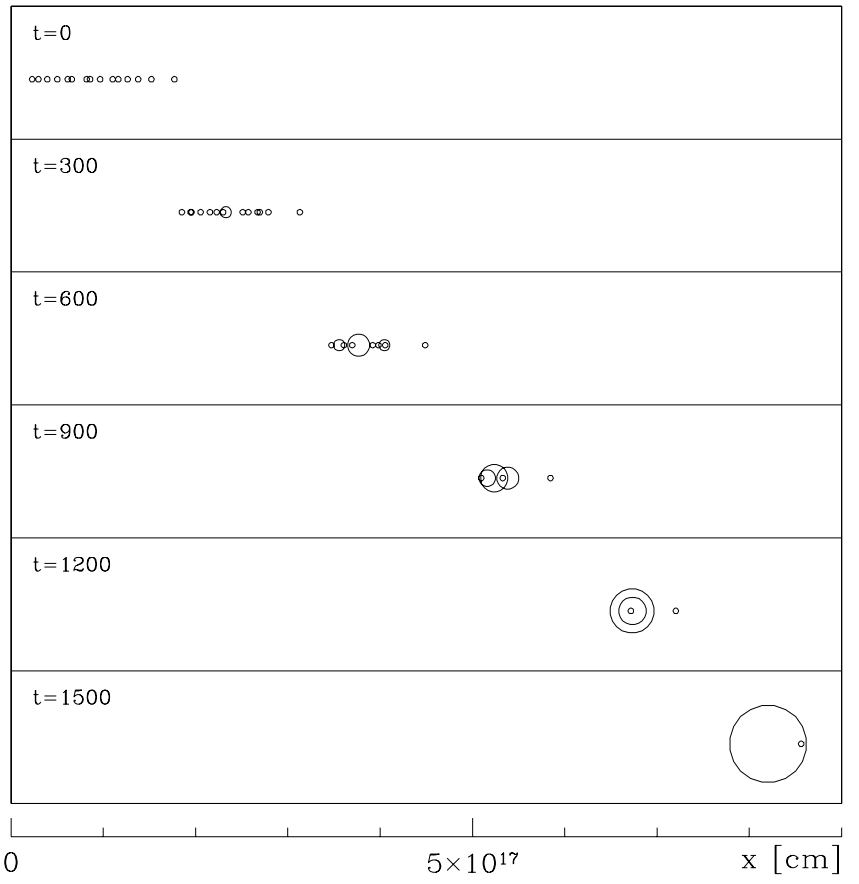

Figure 1. Results from the ballistic, knot-merging model (see Section 2). The top frame shows the present-day positions along the $\mathrm{HH} 34$ jet, and the following frames show future knot configurations (at intervals of $300 \mathrm{yr}$, with the times in years shown on the top left of each frame). By $t=1500 \mathrm{yr}$ all but one of the knots have merged into a massive structure. The radii of the circles (centered on the positions of the successive knots) are proportional to the knot masses. The outflow source is located at the left edge of the frame, and the abscissa (labeled in centimeter at the bottom) is parallel to the outflow axis.

measured the plane-of-the-sky axial velocity of the knots, and $\phi=35^{\circ}$ is the orientation angle derived by Raga et al. 2011c). In this table, we have not included three knots for which Raga et al. (2011c) obtain unreliable proper motions. The 414 pc distance to the Orion cloud obtained by Menten et al. (2007) has been used.

We now assume that the knots move ballistically, so that the distance $x_{k}$ from the source to knot $k$ on the plane of the sky is given by

$$
x_{k}(t)=x_{k, 0}+v_{k} t,
$$

where $x_{k, 0}$ is the present position (second column of Table 1) and $v_{k}=v_{j} \cos \phi\left(v_{j}\right.$ given in the third column of Table 1) the $x$-velocity of knot $k$.

As the plane-of-the-sky velocities $v_{k}$ of the knots are not identical, knot collisions are likely to occur. Therefore, in order to track the time evolution of the knot structure it is necessary to have a "knot merging law." The straightforward way to specify such a law is to consider that a knot " $q$ " formed through the collision of two knots of masses $m_{l}$ and $m_{k}$ will have a mass

$$
m_{q}=m_{l}+m_{k}
$$

and a plane-of-the-sky velocity

$$
v_{q}=\frac{m_{l} v_{l}+m_{k} v_{k}}{m_{l}+m_{k}},
$$

which follow from mass and momentum conservation.

In order to follow the time evolution of the present-day $\mathrm{HH} 34$ jet knot configuration, we first assign identical masses $m_{k}=1$ to all of the knots of Table 1. We then follow the time evolution assuming ballistic trajectories for the knots (Equation (1)) and applying the knot merging law (Equations (2) and (3)) when
Table 1

Proper Motions of the HH 34 Jet Knots

\begin{tabular}{lccr}
\hline \hline Knot $^{\mathrm{a}}$ & $\begin{array}{c}x \\
\left(10^{16} \mathrm{~cm}\right)^{\mathrm{b}}\end{array}$ & $\begin{array}{c}v_{j} \\
\left(\mathrm{~km} \mathrm{~s}^{-1}\right)^{\mathrm{c}}\end{array}$ & $\begin{array}{c}-t \\
(\mathrm{yr})^{\mathrm{d}}\end{array}$ \\
\hline 3 & 2.22 & 207 & 42 \\
4 & 2.90 & 212 & 53 \\
5 & 3.86 & 201 & 75 \\
6 & 4.94 & 200 & 96 \\
8 & 6.04 & 198 & 119 \\
9 & 6.49 & 202 & 125 \\
10 & 8.09 & 190 & 165 \\
11 & 8.47 & 190 & 173 \\
12 & 9.55 & 174 & 213 \\
13 & 10.89 & 181 & 234 \\
14 & 11.49 & 181 & 247 \\
15 & 12.50 & 182 & 268 \\
16 & 13.64 & 170 & 313 \\
17 & 15.06 & 164 & 359 \\
18 & 17.54 & 174 & 390 \\
$34 S^{-}$ & 65.2 & 298 & 848 \\
$34 S^{+}$ & 65.2 & 186 & 1364 \\
\hline
\end{tabular}

Notes.

a The successive knots away from the source are numbered consecutively.

b $x$ is the offset from the position of the source measured along the outflow axis in the 2007.83 frame. The angular separations have been converted into centimeter assuming a $414 \mathrm{pc}$ distance to $\mathrm{HH} 34$.

c Velocities along the outflow axis assuming a $414 \mathrm{pc}$ distance.

d Time of knot ejection computed as $t=-x / v_{x}$.

knot collisions occur. The knots are assumed to have vanishingly small sizes.

The resulting, time-dependent knot structure is shown in Figure 1. In the top panel, we see the $t=0$, present-day configuration of knots 3-18. The other panels show future configurations. The knots are shown with circles centered at the knot position, with radii $r_{k} \propto m_{k}$. Therefore, knots resulting from mergers of many knots have larger radii (reflecting their larger masses). For example, at $t=1500 \mathrm{yr}$ (bottom frame of Figure 1), we have only two knots, one with a unitary mass (the leading knot of the initial chain, which has had no collisions) and a second one with a mass of 14 (resulting from the merger of all of the other knots).

From Figure 1, it is clear that by $t=900 \mathrm{yr}$ most of the knots have merged, and that the merged knots are converging on a spatially limited region. The resulting mass accumulation is located at $x \approx 5.3 \times 10^{17} \mathrm{~cm}$. By $t=1200 \mathrm{yr}$, the mass accumulation region has reached $x \approx 6.8 \times 10^{17} \mathrm{~cm}$, which is close to the present-day position of the $\mathrm{HH} 34 \mathrm{~S}$ bow shock (with position $x_{34 S} \approx 6.6 \times 10^{17} \mathrm{~cm}$ ).

In order to explore whether the mass accumulation that will be formed by the present $\mathrm{HH} 34$ jet knots will in fact resemble the HH 34S bow shock, we carry out gas-dynamical simulations. These are described in the following section.

\section{GAS-DYNAMICAL SIMULATIONS}

\subsection{The Ejection Velocity Time Variability}

In order to carry out a full gas-dynamical simulation of the $\mathrm{HH}$ 34 outflow, it is necessary to specify the full time dependence of the ejection velocity. However, the observed motions of the knots along the outflow determine the ejection history only in a partial way.

This is seen in Figure 2, where we show the spatial velocities of the HH 34 jet knots (obtained as $v_{j}=v_{T} / \cos \phi$, where 


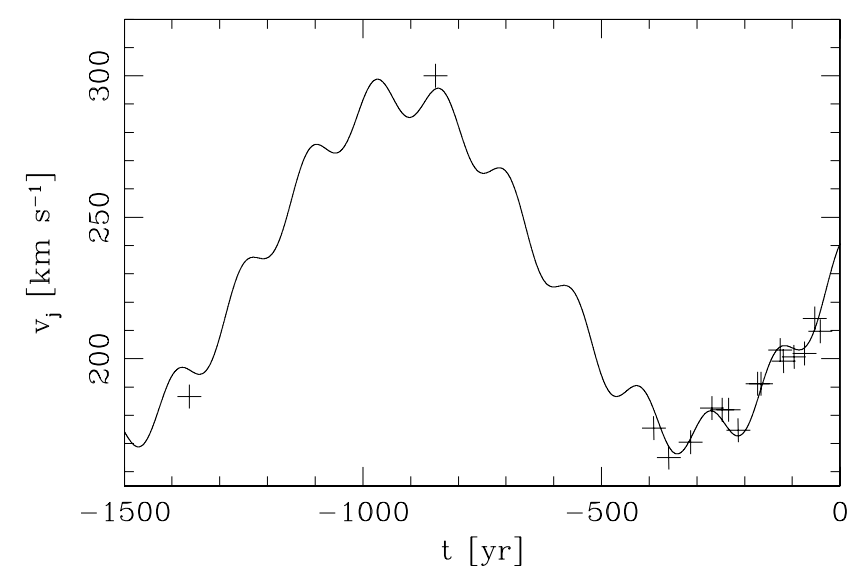

Figure 2. Ejection velocity vs. time reconstructed from the positions and velocities given in Table 1. The solid line shows the results of the two-mode sinusoidal fit described in Section 3.1.

$\phi=35^{\circ}$ is the orientation angle of the outflow axis deduced by Raga et al. 2011c) against the time

$$
t=-\frac{x}{v_{x}}
$$

at which they were ejected (i.e., assuming that the motions are ballistic).

In Figure 2, we have also added the dynamical times and jet velocities deduced by Raga \& Noriega-Crespo (1998) for the material directly upstream and downstream of the $\mathrm{HH} 34 \mathrm{~S}$ bow shock (see the entries labeled $34 \mathrm{~S}^{-}$and $34 \mathrm{~S}^{+}$in Table 1 ) from the position-velocity diagram of Heathcote \& Reipurth (1992). These two points together with the ones deduced for the HH 34 jet knot chain provide constraints on part of the ejection velocity history of this outflow.

To the $v_{j}(t)$ points deduced from the data, we have carried out a least-squares fit of a double-sinusoidal mode function of the form:

$$
v_{j}(t)=v_{0}+v_{1} \sin \left(\frac{2 \pi t}{\tau_{1}}+\phi_{1}\right)+v_{2} \sin \left(\frac{2 \pi t}{\tau_{2}}+\phi_{2}\right),
$$

obtaining $v_{0}=231 \mathrm{~km} \mathrm{~s}^{-1}, v_{1}=60 \mathrm{~km} \mathrm{~s}^{-1}, \tau_{1}=1227 \mathrm{yr}, \phi_{1}=$ $0.001 \mathrm{rad}, v_{2}=8 \mathrm{~km} \mathrm{~s}^{-1}, \tau_{2}=143 \mathrm{yr}$, and $\phi_{2}=1.225 \mathrm{rad}$. This fit is shown in Figure 2. We have computed an axisymmetric numerical simulation of a jet with this ejection time variability.

We have also computed a three-mode model in which we include a third mode of the form $v_{3} \sin \left(2 \pi t / \tau_{3}\right)$ with $v_{3}=$ $10 \mathrm{~km} \mathrm{~s}^{-1}$ and $\tau_{3}=27 \mathrm{yr}$. Raga \& Noriega-Crespo (1998) argued that such a mode is necessary for producing the aligned knots along the $\mathrm{HH} 34$ jet.

\subsection{The Models}

We have computed a two-mode (model "a") and a threemode (model "b") simulation with the time-dependent ejection velocities described in Section 3.1. For both models, we have considered a time-independent injection density $n_{j}=10^{3} \mathrm{~cm}^{-3}$ and temperature $T_{j}=1000 \mathrm{~K}$, and a $r_{j}=5 \times 10^{15} \mathrm{~cm}$ initial jet radius. The environment is initially homogeneous, with a density $n_{\text {env }}=15 \mathrm{~cm}^{-3}$ and temperature $T_{\text {env }}=1000 \mathrm{~K}$. Both the jet and the environment are initially neutral, with a seed electron density coming from singly ionized carbon.

Models "a" and "b" (see above) have a top-hat initial cross section. We have also computed a third simulation (model "c"), with the same three-mode variability as model "b," but with a parabolic injection velocity cross section with a $\sigma=0.75$ edge-to-center velocity ratio. Model "c" was computed with an $r_{j}=2 \times 10^{15} \mathrm{~cm}$ initial jet radius.

The numerical setup is identical to that of Raga et al. (2011a). A cylindrical domain of $(16.8,2.1) \times 10^{17} \mathrm{~cm}($ axial $\times$ radial $)$ is resolved with a six-level (models " $a$ " and "b") or seven-level (model "c"), binary adaptive grid of $4.1 \times 10^{14} \mathrm{~cm}$ (models "a" and "b") or $2.1 \times 10^{14} \mathrm{~cm}$ (model "c") maximum resolution along the two axes. An inflow condition is applied in the $r \leqslant r_{j}$ region of the $z=0$ boundary and a reflection condition for $r>r_{j}$. A reflection condition is applied on the symmetry axis, and transmission conditions on the remaining grid boundaries. The gas-dynamic equations are integrated together with an ionization network including $\mathrm{H}, \mathrm{He}, \mathrm{C}, \mathrm{N}, \mathrm{O}$, and $\mathrm{S}$ ions, which are used to compute the energy-loss term which is included in the energy equation (for details, see Raga et al. 2011a), using the “yguazú-a” code (Raga et al. 2000).

\subsection{The Resulting Jet Structures}

We have run the three simulations described in Sections 3.1 and 3.2, turning on the jet flows at a time $t=-2500 \mathrm{yr}(t=0$ corresponding to the present time), and letting them evolve until $t=0$. From the resulting density, electron density, temperature, and ionization state, we compute [S II] 6716+30 emission maps, which can be compared directly with present-day observations of $\mathrm{HH} 34$.

In Figure 3, we show a comparison between the 1998.71 HST [S II] image of HH 34 and the corresponding maps predicted (for $t=0$, see above) from models "a," "b," and "c." For the calculation of the [S II] intensity maps we have assumed a $\phi=35^{\circ}$ orientation angle between the outflow axis and the plane of the sky (as suggested by Raga et al. 2011c).

It is clear that the three models produce a large bow shock, with a qualitative similarity to $\mathrm{HH} 34 \mathrm{~S}$. In spite of this general similarity, the predicted bow shock is $\sim 20 \%$ further away than $\mathrm{HH} 34 \mathrm{~S}$ in all models (see Figure 3).

Also clear from Figure 3 is that the two-mode model "a" does not have a chain of aligned emitting knots such as observed in $\mathrm{HH} 34$. This is due to the fact that the second mode (see Equation (5)) does not have a large enough amplitude to produce shocks with substantial [S II] emission. For producing a chain of knots, it is therefore necessary to introduce the third mode (see the text following Equation (5)) suggested by Raga \& Noriega-Crespo 1998, which does result in a chain of emitting knots.

A comparison of the observed chain of knots and the predictions of models " $b$ " and "c" is shown in Figure 4. It is clear that the introduction of an axially peaked ejection velocity cross section (model "c") produces quite dramatic changes in the predicted intensities and morphologies of the knot emission (this effect was discussed by Raga et al. 2011a). Model "c" produces a chain of knots with better qualitative resemblance to the HH 34 jet knots (see Figure 4).

\section{SUMMARY AND CONCLUSIONS}

The proper motions for the knots along the HH 34 jet derived by Raga et al. (2011c) show decreasing velocities as a function of increasing distances from the outflow source. If we project the motions into the future, we therefore find that the knots will merge into a structure with the combined masses of the present-day knots. 
$10^{-3}$

$10^{-2}$
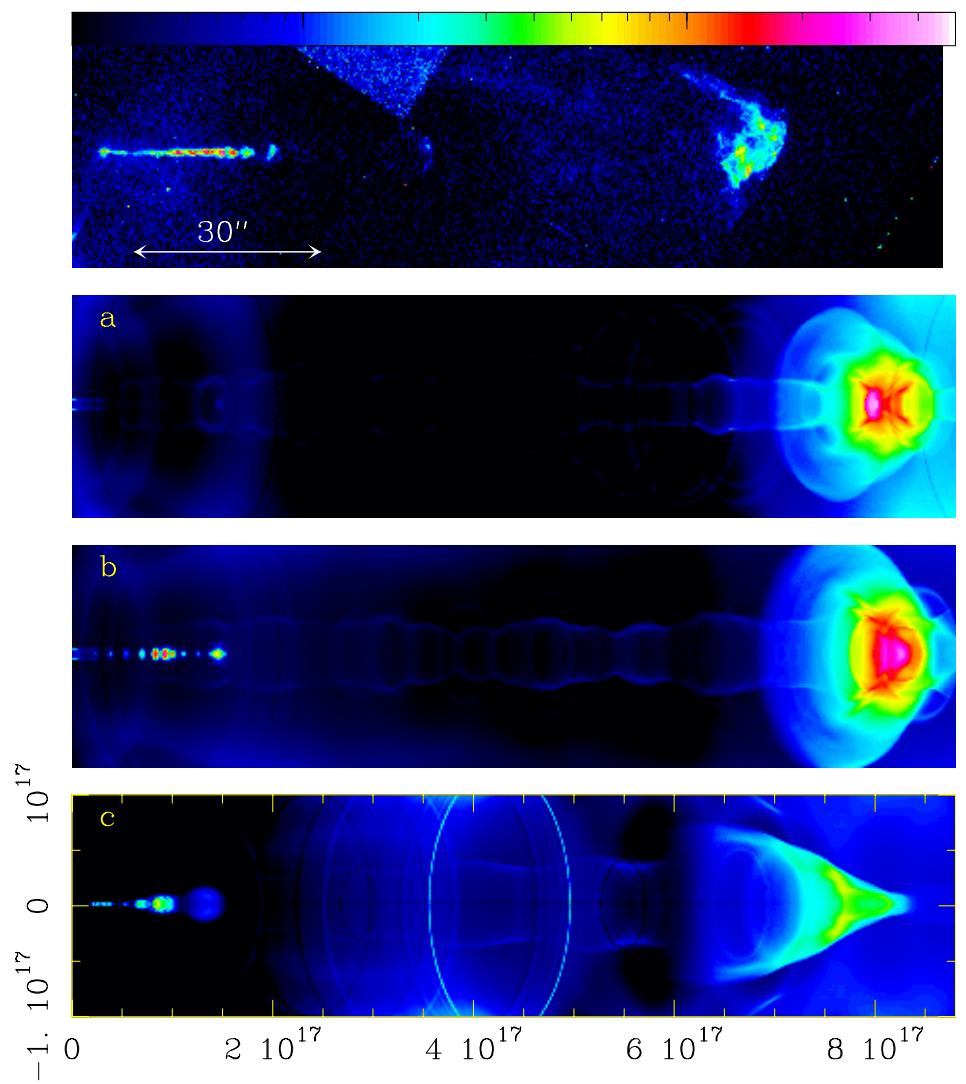

Figure 3. HST [S II] 6716+30 image of HH 34 (top, rotated so that the outflow axis is approximately parallel to the abscissa) and the corresponding intensity maps from models "a," "b," and "c" described in Section 3.2. The flux of the HH 34 image has been multiplied by a factor of 1/100 so as to have intensities comparable to the emission from the models. The logarithmic color scale is given (in $\mathrm{erg} \mathrm{cm}^{-2} \mathrm{~s}^{-1} \mathrm{sr}^{-1}$ ) by the top bar. The HH 34 image has been placed on the same spatial scale as the model predictions assuming a $414 \mathrm{pc}$ distance. The axes are labeled in centimeter in the bottom frame.

(A color version of this figure is available in the online journal.)

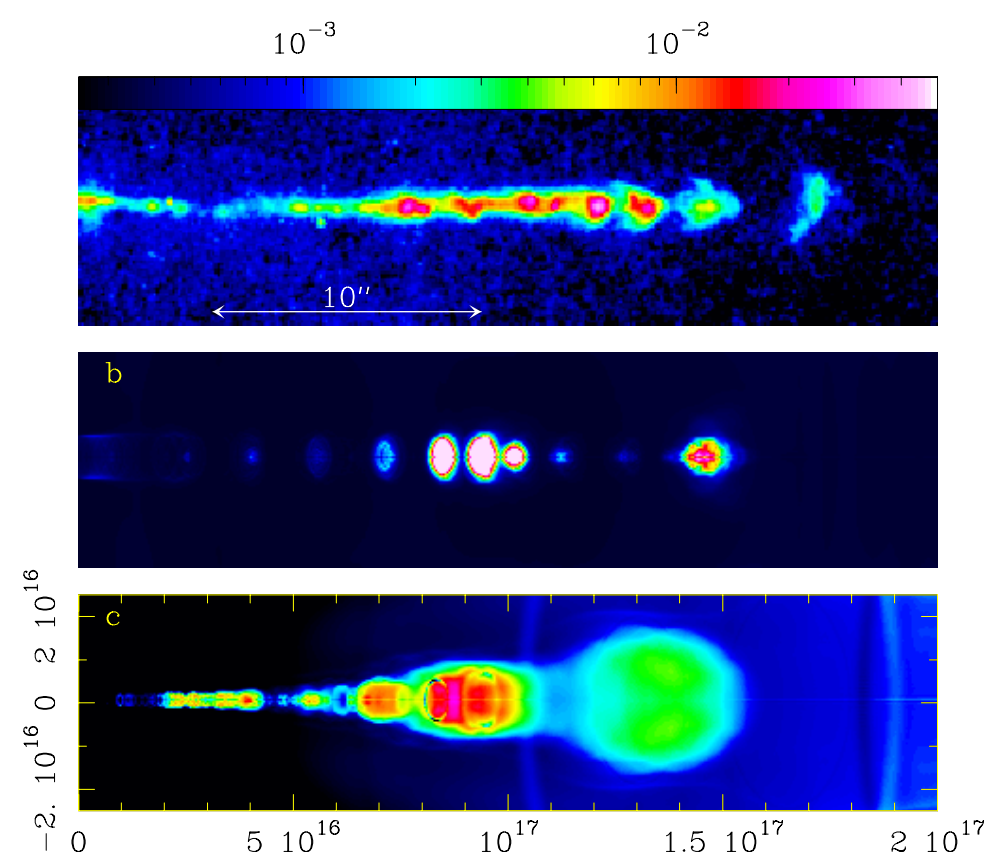

Figure 4. Zoom of the region close to the outflow source of the [S II] emission of HH 34 (top) and the predicted emission from models "a" (center) and "b" (bottom) The intensity of $\mathrm{HH} 34$ has been multiplied by a factor of 1/100, and the map predicted from model "b" by a factor two. The logarithmic color scale is given (in erg cm $\mathrm{cm}^{-2} \mathrm{~s}^{-1} \mathrm{sr}^{-1}$ ) by the top bar. The HH 34 image has been placed on the same spatial scale as the model predictions assuming a $414 \mathrm{pc}$ distance.

(A color version of this figure is available in the online journal.) 
We have modeled this merging process considering ballistic motions for the knots and a mass and momentum conservation "knot merging law." We find that most of the knots will combine into a single structure in $\sim 1000 \mathrm{yr}$, and that this structure will be formed at $\sim 6 \times 10^{17} \mathrm{~cm}$ from the outflow source (projected on the plane of the sky), agreeing well with the present-day position of the HH 34S bow shock. This "knot merging model" is described in Section 2.

We have also made a two-mode sinusoidal fit to the ejection velocity time history reconstructed from the proper motions of Raga et al. (2011c) and from the radial velocities of the HH $34 \mathrm{~S}$ region (following Raga \& Noriega-Crespo 1998). We have also added the third, higher frequency mode suggested by Raga \& Noriega-Crespo (1998) in order to form the observed HH 34 jet knots (see Section 3.1). We have computed axisymmetric simulations with a "top hat" initial cross-section, and with an initial cross-section with a parabolic velocity profile with a $\sigma=0.75$ edge-to-center velocity ratio (following the suggestion of Raga et al. 2011a). We find that the $\sigma=0.75$, three-mode model produces the "synthetic jet" with the best qualitative agreement yet with the observed [S II] emission of the knots along the $\mathrm{HH} 34$ jet. That this is indeed the case can be seen by checking the previous model predictions of Raga \& Noriega-Crespo (1998) and Raga et al. (2002, 2011a), which were calculated with somewhat different ejection velocity time dependencies.

Clearly, it would be interesting to see whether or not our "best model" (model "c," see Sections 3.2 and 3.3) reproduces other observational characteristics of the HH 34 outflow. Obvious possibilities are: intensity maps in other emission lines, spatially resolved line ratios, and spatially resolved emission line profiles (both optical and IR). In order to make meaningful comparisons with the observations, it will of course be necessary to compute simulations with considerably higher numerical resolutions (in the present simulations, the initial jet radius is resolved with only $\sim 10$ points, see Section 3.2).

Such comparisons between model predictions and observations of $\mathrm{HH} 34$ are likely to show that the models are still missing relevant components. We would judge this work successful if it turns out that our present models do indeed include some of the main ingredients necessary for explaining the dynamical properties of the $\mathrm{HH} 34$ jet.

We end by noting that some theoretical studies (Roberts 1986; Raga 1992; Yirak et al. 2009; Bonito et al. 2010a, 2010b) have studied the formation of knot structures as a result of successive collisions between smaller knots ejected randomly by the source. However, in the knots along the HH 34 jet we observe a general trend of increasing ejection velocities at more recent times, which is inconsistent with a purely random knotto-knot ejection variability.

Some of the present-day knots could of course be the result of mergers of smaller structures. However, unless we are prepared to assume that in the region close to the outflow source there is a large reservoir of small, undetected knots, we are forced to conclude that such mergers are rather infrequent in the past in the knots along the HH 34 jet. However, it is clear that the present-day knots will have a rather spectacular succession of knot merging events $\sim 1000$ yr from now.

This conclusion is based on the assumption that the motions of the knots do not differ substantially from ballistic motions. If the observed knots correspond to internal working surfaces (produced by an ejection variability), they should indeed move at a constant velocity (until they collide with other knots), as internal working surfaces rapidly achieve a time-independent velocity (demonstrated analytically by Cantó et al. 2000). Actually, following the discovery of a counterjet with remarkable knot-to-knot symmetry in HH 34 (García López et al. 2008; Raga et al. 2011b) there is now little doubt that this model is indeed applicable for the HH 34 outflow.

The variability implied by the proper motion measurements of HH 34 could correspond to the orbital period of a binary source in an elliptical orbit (see Reipurth 2000; Reipurth et al. 2010). Unfortunately, there are no theoretical models of the effect of the close passage of a companion on the production of an outflow from a star+accretion disk system. Interestingly, Witt et al. (2009) report observations of the Red Rectangle protoplanetary nebula showing that higher outflow velocities occur close to the periastron of the highly excentric binary source. Possibly, similar effects might be revealed by future observations of outflows from young stars.

Based on observations made with the NASA/ESA Hubble Space Telescope, and obtained from the Hubble Legacy Archive, which is a collaboration between the Space Telescope Science Institute (STScI/NASA), the Space Telescope European Coordinating Facility (ST-ECF/ESA), and the Canadian Astronomy Data Centre (CADC/NRC/CSA). The work of A.R., A.R.G., and A.E. was supported by the CONACyT grants 61547 , 101356, and 101975. We thank John Bally (the referee) for helpful comments.

\section{REFERENCES}

Bally, J., \& Devine, D. 1994, ApJ, 428, L65

Bonito, R., Orlando, S., Peres, G., et al. 2010a, A\&A, 511, 42 Bonito, R., Orlando, S., Peres, G., et al. 2010b, A\&A, 517, 68 Bührke, T., Mundt, R., \& Ray, T. P. 1988, A\&A, 200, 99

Cantó, J., Raga, A. C., \& D’Alessio, P. 2000, MNRAS, 313, 656

Devine, D., Bally, J., Reipurth, B., \& Heathcote, S. 1997, AJ, 114, 2095

Eislöffel, J., \& Mundt, R. 1992, A\&A, 263, 292

García López, R., Nisini, B., Giannini, T., et al. 2008, A\&A, 487, 1019

Hartigan, P., Frank, A., Foster, J. M., et al. 2011, ApJ, 736, 29

Heathcote, S., \& Reipurth, B. 1992, AJ, 104, 2193

Herbig, G. H. 1974, LicOB, 658, 1

Masciadri, E., de Gouveia Dal Pino, E. M., Raga, A. C., \& Noriega-Crespo, A. 2002, ApJ, 580, 950

Menten, K. M., Reid, M. J., Forbrich, J., \& Brunthaler, A. 2007, A\&A, 474, 515

Morse, J. A., Hartigan, P., Cecil, G., Raymond, J. C., \& Heathcote, S. 1992, ApJ, 399, 231

Podio, L., Bacciotti, F., Nisini, B., et al. 2006, A\&A, 456, 189

Raga, A. C. 1986 , AJ, 92, 637

Raga, A. C. 1992, MNRAS, 258, 301

Raga, A. C., Navarro-González, R., \& Villagrán-Muniz, M. 2000, RevMexAA, 36, 67

Raga, A. C., \& Noriega-Crespo, A. 1998, AJ, 116, 2943

Raga, A. C., Noriega-Crespo, A., Kajdic, P., et al. 2011a, RMxAA, 47, 277

Raga, A. C., Noriega-Crespo, A., Lora, V., Stapelfeldt, K. R., \& Carey, S. J. 2011b, ApJ, 730, L17

Raga, A. C., Noriega-Crespo, A., Rodríguez-González, A., et al. 2011c, AJ, submitted

Raga, A. C., Velázquez, P. F., Cantó, J., \& Masciadri, E. 2002, A\&A, 395, 647

Ray, T. P., Mundt, R., Dyson, J. E., Falle, S. A. E. G., \& Raga, A. C. 1996, ApJ, 468, L103

Reipurth, B. 2000, AJ, 120, 3177

Reipurth, B., Bally, J., Graham, J. A., Lane, A. P., \& Zealy, W. J. 1986, A\&A, 164,51

Reipurth, B., \& Heathcote, S. 1992, A\&A, 257, 693

Reipurth, B., Heathcote, S., Morse, J., Heathcote, S., \& Bally, J. 2002, AJ, 123, 362

Reipurth, B., Mikkola, S., Connelley, M., \& Valtonen, M. 2010, ApJ, 725, L56 Roberts, D. A. 1986, ApJ, 300, 568

Witt, A. N., Murthy, J., Gustafson, B., et al. 2009, ApJ, 693, 1946

Yirak, K., Adam, F., Cunningham, A. J., \& Mitran, S. 2009, ApJ, 695, 999 